\title{
PRACTICE OBSERVED
}

\section{Practice Research}

\section{Feasibility of a large prospective study in general practice: an Italian experience}

\author{
GRUPPO DI STUDIO SULLA PRESSIONE ARTERIOSA NELL'ANZIANO*
}

\begin{abstract}
A study of blood pressure control in elderly outpatients was carried out with the participation of 444 Italian general practitioners. Of 4096 patients aged 65 years or over who were considered for recruitment, $3959(96.7 \%)$ fulfilled all the criteria of admission and were followed up for 12 months. The findings regarding one of the aims of the study-that is, to assess the feasibility of a large scale trial in general practice-are reported. Most $(87 \%)$ of the doctors completed the study. Their adherence to the protocol was highly satisfactory, leading to an acceptable quality of work. Patients' compliance was also good; $98.6 \%$
\end{abstract}

Istituto di Ricerche Farmacologiche Mario Negri, Milano, Italy

FAUSTO AVANZINI, MD, research fellow

CLAUDIO ALLI, MD, research fellow

GIUSEPPE BETTELLI, MD, research fellow

FABIO COLOMBO, PHD, senior investigator

LUCA CONFORTI, MD, research fellow

FLORINDO PIRONE, MD, research fellow

ALBERTO SPAGNOLI, MD, senior investigator

EMANUELA TAIOLI, MD, research fellow

GIANNI TOGNONI, MD, chief of the laboratory of clinical pharmacology

MASSIMO VILLELLA, MD, research fellow

ALESSANDRO ZUSSINO, MD, research fellow

Istituto di Scienze Biomediche Bassini, Universita' di Milano, Italy

MARCO DI TULLIO, MD, research fellow

GIANCARLO MARIOTTI, $M D$, research registrar

MARIA RADICE, MD, research registrar

Participating doctors: The names of the $\mathbf{4 4 4}$ general practitioners were published in F Avanzini, C Alli, A Andreani, et al. SPAA: studio sulla pressione arteriose in pazienti anziani ambulatoriali. Practitioner (Edizione Italiana) 1984;75:74-83.

Correspondence to: Dr F Avanzini, Istituto di Ricerche Farmacologiche Mario Negri, Via Eritrea 62, 20157 Milano, Italy.

^Coordinating group.
(3898) of the patients who had fulfilled the admission criteria agreed to participate in the study, and only 4\%(158) dropped out.

Both of these observations support the feasibility of carrying out prospective studies in general practice. The creation of networks of general practitioners who are prepared to carry out research in their practices would allow treatment and preventive measures to be studied simply and at low cost in the appropriate setting.

\section{Introduction}

General practice has long been considered the ideal place to carry out research aimed at explaining problems that are normally dealt with under uncontrolled conditions. ${ }^{1-6}$ Despite much urging, a few studies only have dealt with large populations and these mainly in the United Kingdom, where research in general practice is part of an established tradition.

We report the first experience in Italy of a large scale prospective study of blood pressure control in elderly patients (Studio sulla Pressione Arteriosa nell' Anziano), where over 400 general practitioners recruited and reliably followed up a cohort of roughly 4000 patients. The study produced a wealth of information on epidemiology and on the quality of care in the treatment of hypertension in elderly patients, which is still controversial. It also showed that a network of research oriented general practitioners could be established that might pave the way for simpler and low cost ways of assessing treatment and preventive measures under controlled conditions.

\section{Organisation of the study}

The main aim of the study was to assess the prevalence and the degree of control of hypertension in an ambulatory population and to evaluate prospectively the clinical course of the hypertension under uncontrolled 
conditions in general practice. Besides providing such basic epidemiological information, it was stipulated that the study should be seen as a feasibility exercise for a randomised controlled trial on the efficacy of different treatments on the same population or in some selected subgroups.

General practitioners were recruited as volunteers, with no financial incentives such as reimbursements, as an expression of their interest in what is virtually an open question in daily practice. With the support of the Italian Federation of Physicians a letter was sent to provincial representatives asking for "sensitisation" campaigns in their areas. The research protocol was then published in the Italian edition of the Practitioner, which is sent free of charge to all general practitioners in the country. ${ }^{7}$ The general practitioners also received an application form for the study which they had to mail to the coordinating group.

Some 750 general practitioners who expressed interest in the project were divided into provincial groups led by local coordinators who were invited to

\section{Results}

A total of 752 general practitioners agreed to participate in the study and of these, $444(59 \%)$ started the research. These doctors $(396$ men and 48 women) represented $0.7 \%$ of all Italian general practitioners registered at the beginning of the study. Though they were not located uniformly throughout the country, all regions of Italy except Val d'Aosta were represented (fig 1). The highest rate of participation was in northern Italy (287 (64.6\%) doctors), followed by central $(103,23 \cdot 2 \%)$ and southern Italy $(54,12 \cdot 2 \%)$. The mean age of the doctors was 34.5 years, range 25 to 68 years; $334(75 \cdot 2 \%)$ were under 35 (nationally $32 \cdot 9 \%$ ); $196(44 \cdot 1 \%)$ had a specialisation and $56(12 \cdot 6 \%)$ also worked in a hospital. The average number of health service patients who were registered with each participating general practitioner was 1170 (range 55 to 3544), higher than the national figure of 912 . Table I gives the doctors' characteristics by age group.

TABLE I-Characteristics of the general practitioners who participated in the study (percentages in parentheses)

\begin{tabular}{|c|c|c|c|c|}
\hline & \multicolumn{3}{|c|}{ Age group (years) } & \multirow[b]{2}{*}{ Total No (\%) } \\
\hline & $25-34$ & $35-49$ & $\geqslant 50$ & \\
\hline \multicolumn{5}{|l|}{ Sex } \\
\hline $\begin{array}{l}\mathbf{M} \\
\mathbf{F}\end{array}$ & $\begin{array}{r}292(65 \cdot 8) \\
42(9 \cdot 5)\end{array}$ & $\begin{array}{r}54(12 \cdot 2) \\
5(1 \cdot 1)\end{array}$ & $\begin{array}{r}50(11 \cdot 3) \\
1(0 \cdot 2)\end{array}$ & $\begin{array}{r}396 \quad(89 \cdot 2) \\
48(10 \cdot 8)\end{array}$ \\
\hline \multicolumn{5}{|l|}{ Specialisation } \\
\hline $\begin{array}{l}\text { No } \\
\text { Yes }\end{array}$ & $\begin{array}{l}206(46.4) \\
128(28 \cdot 8)\end{array}$ & $\begin{array}{ll}23 & (5 \cdot 2) \\
36 & (8 \cdot 1)\end{array}$ & $\begin{array}{ll}19 & (4 \cdot 3) \\
32 & (7 \cdot 2)\end{array}$ & $\begin{array}{l}248(55 \cdot 9) \\
196(44 \cdot 1)\end{array}$ \\
\hline \multicolumn{5}{|c|}{ Also working in hospital } \\
\hline $\begin{array}{l}\text { No } \\
\text { Yes }\end{array}$ & $\begin{array}{r}287(64 \cdot 6) \\
47(10 \cdot 6)\end{array}$ & $\begin{array}{r}53(11 \cdot 9) \\
6(1 \cdot 4)\end{array}$ & $\begin{array}{r}48(10.8) \\
3(0.7)\end{array}$ & $\begin{aligned} 388 & (87 \cdot 4) \\
56 & (12 \cdot 6)\end{aligned}$ \\
\hline \multicolumn{5}{|c|}{ No of health service patients } \\
\hline$\leqslant 500$ & $89(20 \cdot 4)$ & $5(1 \cdot 1)$ & $1(0 \cdot 2)$ & $95(21 \cdot 8)$ \\
\hline $501-1000$ & $84(19 \cdot 3)$ & $12(2 \cdot 8)$ & $4(0.9)$ & $100(22 \cdot 9)$ \\
\hline $1001-1500$ & $89(20.4)$ & $15(3 \cdot 4)$ & $4(0.9)$ & $108(24 \cdot 8)$ \\
\hline$\geqslant 1500$ & $65(14 \cdot 9)$ & $26(6 \cdot 0)$ & $42(9 \cdot 6)$ & $133(30 \cdot 5)$ \\
\hline Total & $334(75 \cdot 2)$ & $59(13 \cdot 3)$ & $51(11 \cdot 5)$ & $444(100)$ \\
\hline
\end{tabular}

${ }^{\star}$ Missing data for eight.

attend a meeting in Milan and a meeting in Rome in December 1982. The study protocol was presented and discussed, and local coordinators were trained to check the reliability of the sphygmomanometers and to monitor problems that might arise during routine application of the protocol.

General practitioners who confirmed their willingness to participate in the trial were asked to have their sphygmomanometers checked by the local coordinator and to recruit two model patients, whose cases were analysed and discussed at 29 meetings held in the various areas before the official recruitment began. This started in March 1983 and was completed 10 months later; $3355(81.9 \%)$ of the patients were enrolled within the first two months (mean (SD) recruitment time per general practitioner was 25 (23) days). The coo:dinating group was available throughout the study for telephone consultations. Each form, sent with a stamped envelope, was checked for completeness and correctness when returned, and any missing details or corrections were asked for.

\section{Methods}

Each participating physician had to recruit at least 10 people aged 65 years or more over a two month period. Patients were chosen randomly from among those who went to the doctors' offices for any reason. The purpose of the study was explained to the patient, and consent was obtained.

Each patient was assessed at two visits at one week's interval and then at four further visits at quarterly intervals to cover the 12 months of planned follow up. On each occasion blood pressure and heart rate were recorded in the sitting position and then after 5 minutes supine and after $\mathbf{3 0}$ seconds standing. During the first visit blood pressure was measured in both arms to identify the one with the higher value, which would be used at later visits. Body weight was recorded. During the initial assessment the presence of main cardiovascular risk factors and the clinical history were recorded, with particular regard to diseases and symptoms associated with hypertension and postural hypotension. Details of drug treatment were taken. During the next visits changes in drug treatment and onset of cardiovascular complications or other noteworthy diseases were investigated. When a patient failed to attend a follow up visit the doctor contacted the patient and noted the reason for the absence. Data were recorded on a form planned for use on a computer; 139 variables were included in the initial assessment, 107 in subsequent visits.
Comparing the participants and the non-participants after initial agreement shows that a higher proportion of the latter were aged over 50 , lived in southern regions, and also worked in a hospital; the average number of health service patients per doctor did not differ between the two groups. Most of the participants ( 389 of $444 ; 87.6 \%$ ) were organised as groups under

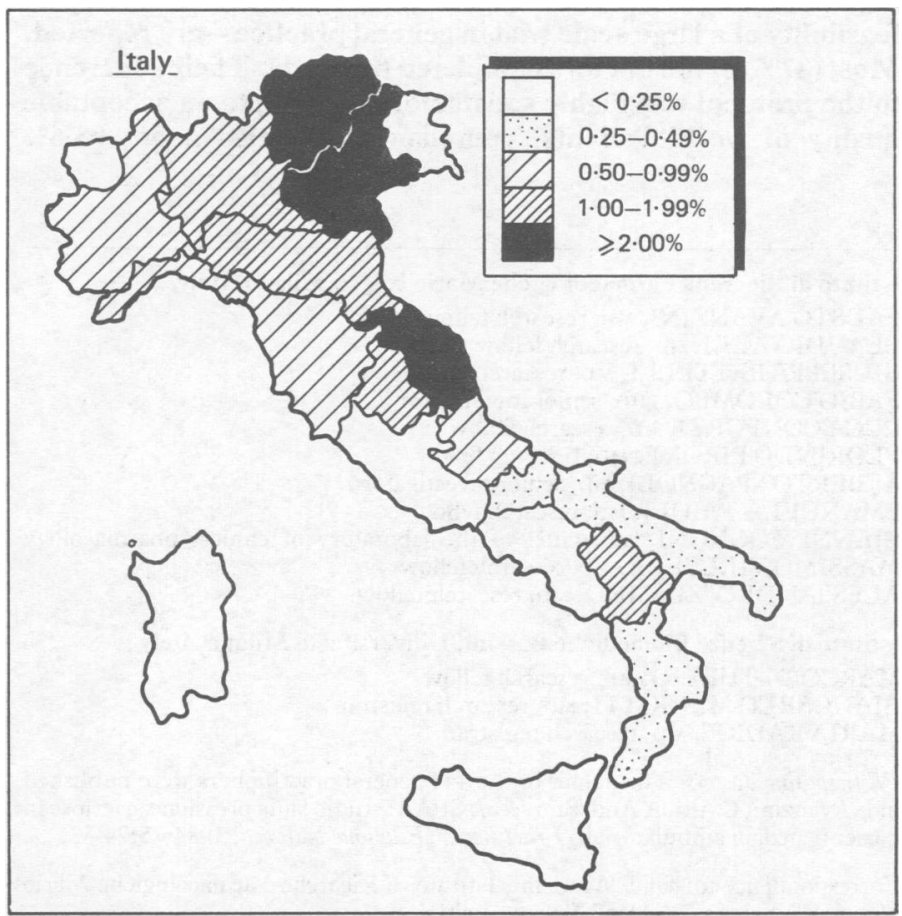

FIG 1-Map of Italy showing the proportion of general practitioners in each region who participated in the study. 
a coordinator, and the remainder participated without a coordinator. Four doctors had coordinating tasks only and eight worked in pairs. Fifty-eight $(13 \cdot 1 \%)$ doctors dropped out of the study, the most common reasons being health problems or changes in working conditions.

Quality of the work-A total of 4096 patients were recruited, $93.9 \%$ of the expected number of 4360 patients. An average of nine patients was recruited by each doctor; in $103(23.6 \%)$ cases the requested number of 10 patients was not reached. Recruitment was correct in $426(97 \cdot 7 \%)$ cases; 10 doctors only were excluded from the study because four did not adhere to the recruitment criteria-for example, they recruited hypertensive patients only-and six did not adhere to the recruitment methods - for example, they did not follow the randomisation order-thus $97(2 \cdot 4 \%)$ patients were excluded. The usefulness of the preliminary phase, which consisted of compiling two trial forms, was assessed by comparing those forms with the first two study forms in a sample of 100 doctors who had been chosen to represent the whole population. The number of serious errors-that is, errors concerning a patient's age, sex, blood pressure measurements, history of cardiovascular diseases, and use of cardiovascular drugs-was halved (from 50 to 23) and minor errors dropped by about $80 \%$ (from 1028 to 202 ).

Table II summarises the quality of the completed forms throughout the study. The number of correct forms gradually rose from $67 \cdot 7 \%$ to $88 \cdot 8 \%$; the
12 month follow up; $111(2.9 \%)$ patients died during the study (fig 3 ). Overall, 606 (15.5\%) patients did not complete the 12 month follow up. A better estimate of patients' non-compliance is given by subtracting the 448 $(11.5 \%)$ patients who could not be included in the follow up because their doctors withdrew from the study. Only $95(2.4 \%)$ patients refused to continue, $55(1.4 \%)$ moved to another town or chose another physician, and eight $(0.2 \%)$ did not attend subsequent visits for unknown reasons. From $0.7 \%$ to $5.3 \%$ of patients did not attend one of the visits because of other reasons, the most common being admission to hospital (fig 2 ). Only 115 patients failed to attend two or more visits consecutively.

\section{Discussion}

The reliable performance of the general practitioners in this study came somewhat as a surprise. Though the protocol included no procedures or knowledge beyond what may be considered good practice, it was nevertheless complex, especially for people who were not used to doing controlled research (the predoctoral curriculum in Italy does not include courses in epidemiology or

TABLE II-Quality of the completed forms used in the study (percentages in parentheses)

\begin{tabular}{|c|c|c|c|c|c|}
\hline & \multirow[b]{2}{*}{ Start } & \multicolumn{4}{|c|}{ Months of study } \\
\hline & & 3 & 6 & 9 & 12 \\
\hline $\begin{array}{l}\text { Forms with: } \\
\text { Serious errors } \\
\text { Minor errors only } \\
\text { No errors } \\
\text { Total No of forms }\end{array}$ & $\begin{aligned} 363 & (9 \cdot 2) \\
915 & (23 \cdot 1) \\
2681 & (67 \cdot 7) \\
3959 & (100)\end{aligned}$ & $\begin{aligned} 53(1 \cdot 4) \\
646(17 \cdot 4) \\
3023(81 \cdot 2) \\
3722(100)\end{aligned}$ & $\begin{aligned} & 51(1 \cdot 5) \\
& 485(13 \cdot 8) \\
& 2970(84 \cdot 7) \\
& 3506(100)\end{aligned}$ & $\begin{aligned} 32 & (1 \cdot 0) \\
326 & (9 \cdot 8) \\
2959 & (89 \cdot 2) \\
3317 & (100)\end{aligned}$ & $\begin{array}{rr}49 & (1 \cdot 5) \\
314 & (9 \cdot 7) \\
2875 & (88 \cdot 8) \\
3238 & (100)\end{array}$ \\
\hline
\end{tabular}

average number of errors or missing data tended to fall (from one every 308 variables to one every 690 variables for serious shortcomings, and from one every 102 variables to one every 311 variables for minor faults). Forty patients were excluded because of serious shortcomings in their initial assessment forms, mainly owing to the doctor choosing the arm with the lower blood pressure for the next measurements at the same visit. After requests for correction or missing data the number of forms still containing one or more errors was low (89 of $17742(0.5 \%)$ with serious errors; 497 of $17742(2 \cdot 8 \%)$ with minor faults).

Patients' adherence to the study-Of the 3959 patients who were admitted after the exclusions mentioned above, only $61(1.5 \%)$ refused to participate. Figure 2 shows the degree of expected $v$ observed attendance at each scheduled visit for the population of patients in the study at each interval. Altogether, 3181 subjects ( $81.6 \%$ of 3898 recruited patients) completed the

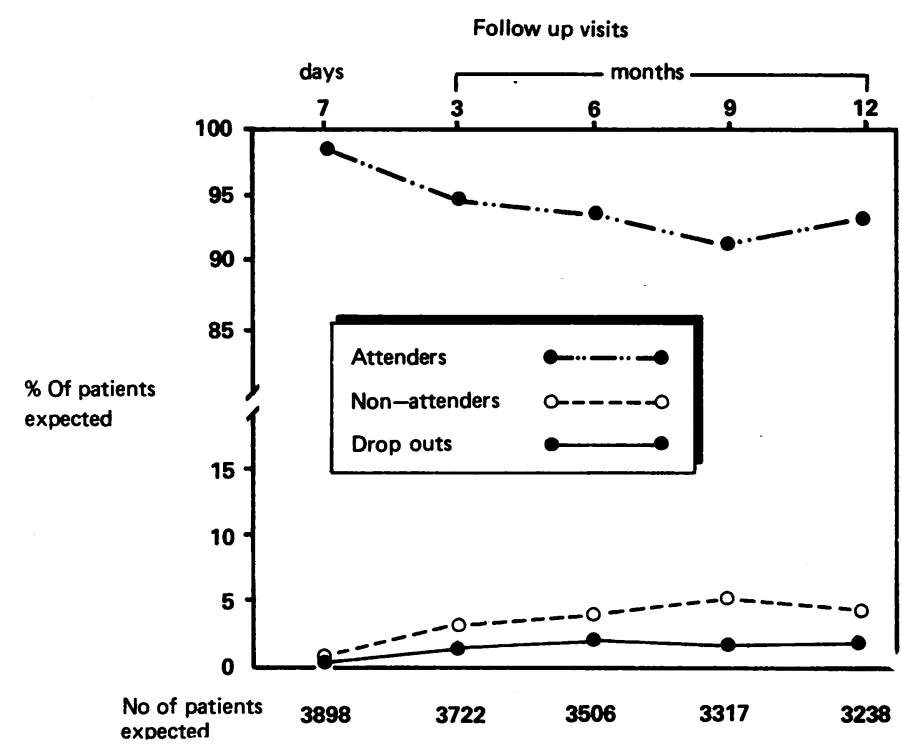

FIG 2-Patients' attendance rates at single follow up visits: the percentage of patients who attended, who did not attend, and who dropped out.

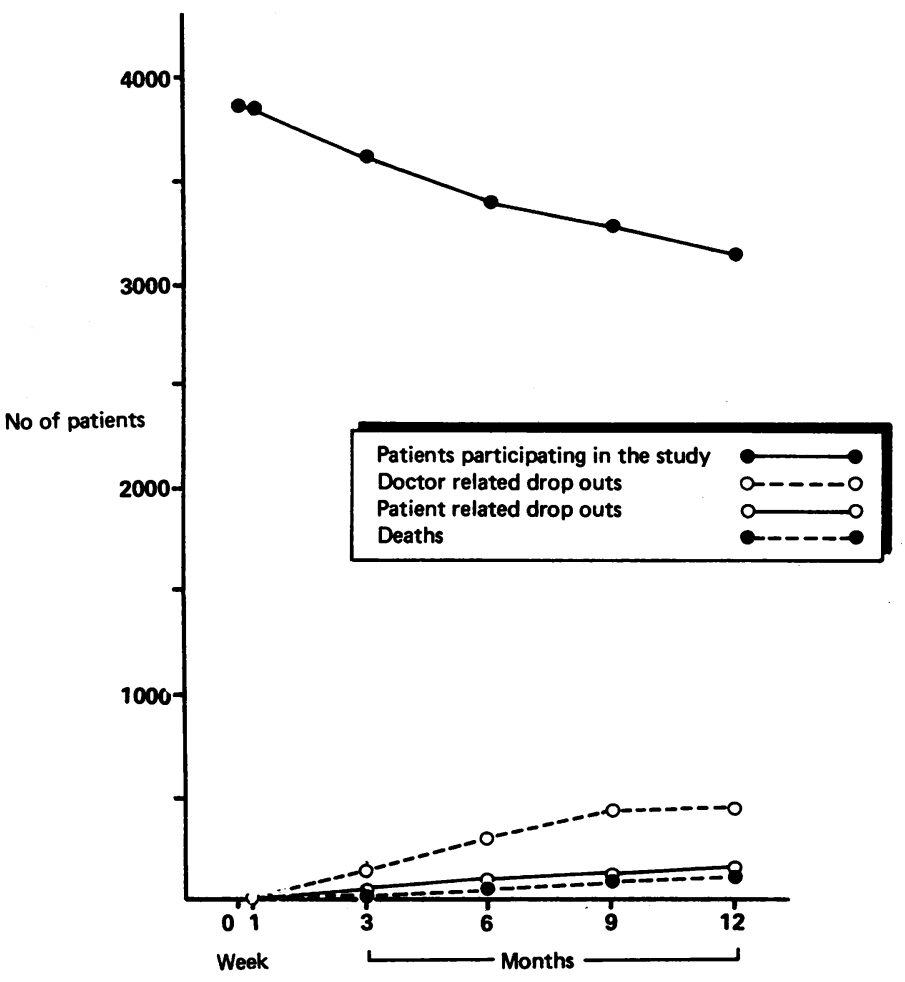

FIG 3-Patients' adherence to the study ( $\mathrm{n}=3898$ patients).

clinical trials). This suggests that when a research protocol fulfils real needs and does not introduce artificial changes into daily practice an appreciable number of general practitioners will be sufficiently motivated to enter a study on scientific grounds. The results are even more encouraging against the background of the health service in Italy, which is usually described as being threatened 
by professional lack of motivation and as being attentive only to contractual conflicts arising from increasing costs and the overcrowding of doctors (currently one per 252 inhabitants).

Perhaps the most convincing proof of the opportunities for worthwhile research in the delivery of health care is that only a small number of general practitioners dropped out. Most of the doctors completed the follow up period, and many have agreed to take part (with a newly recruited group) in a randomised clinical trial of antihypertensive treatments against no treatment in the isolated systolic hypertension of elderly patients. The response of the patients in the study also showed that this section of the population, which is usually exposed to inaccurate prescribing and poor care, ${ }^{8}$ reacts favourably to attention from their general practitioners and has a highly satisfactory rate of compliance.

The fact that there seem to be whole networks of general practitioners who consider formal study protocols to be a natural part of their continuing professional education opens up opportunities for clinical and epidemiological research. Furthermore, because of the combination of a rigorous protocol and attention to the needs of large sections of the population this might be a method for research in clinical pharmacology.'

We do not know of comparable experiences in other health care systems. Repetition of this study or similar studies might be promising in countries where restricted funding of research keeps many doctors from playing a part in developing knowledge and inw testing hypotheses under controlled conditions in general practice. $\vec{z}$

The work was supported by a grant from the National Council of Research (CNR) for the development of clinical pharmacology. We thank the 444 general practitioners who participated; the FNOM (Italian Federation of Physicians) and the Fondazione Angelo and Angela Valenti, Milan, Italy, for generous contributions; Miss Angela Palumbo for data input; and Mrs Maria Nigro for editorial help.

\section{References}

1 Anonymous. Research: the role of the general practitioner [Editorial]. $\boldsymbol{f} R$ Coll Gen Pract 1983;33:469-71.

2 Rakel RE. Family medicine: the need for research. JAMA 1983;249:75-6.

3 Morrell DC. Research in general practice. Problems, priorities, and possibilities. $\mathrm{Br}$ Med $\mathcal{J} \widetilde{\Phi}$ 1982;285:413-4.

4 Falk WA. Research in general practice. Can Med Assoc $\mathcal{F}$ 1979;120:1198-2000.

. Research in general practice. South Afr Med J 1981;59:296-8. Anymous. Trials in general practice. In: Hersheimer A, Zentler-Munro P, Winn D, eds. Therapeutic trials and society. Making the best use of resources. London: Consumers' Association,: 1986:9.

7 Tognoni G, Avanzini F, Coen D, Liberati A, Spegnoli A. Formazione e ricerca nella pratica di $\vec{\omega}$ medicina generale. Una proposta. Practitioner (Edisione Italiana) 1982;54:53-64.

8 World Health Organisation. The control of drugs for the elderty. Copenhagen: WHO, 1981. (Euro Report No 50.)

9 Bonati M, Togmoni G. Has clinical pharmacology lost its way? Lancet 1984;i:556-8.

(Accepred 28 July 1986)

\section{Multicultural medicine}

Indian six week rule-A British general practitioner was surprised when a young Indian mother with her first born child after his check up at six weeks said, "I am so pleased, doctor, because today he is 6 weeks old, and I can now dress him in new clothes." When the doctor asked her, "Why not before now?" she told him that according to centuries old Indian tradition a mother is not supposed to buy her baby new clothes for six weeks after birth and that she has to borrow old clothes from relatives or friends. She could not give any reason for this and did not know anyone else who could. I suggest that this could be because neonatal deaths were so common in poor Indian villages that new clothes were considered not to be worth buying until the baby's hold on life was firmly established.

Another Indian tradition should also be remembered. A new mother is not supposed to take her baby out for $\mathbf{4 0}$ days, possibly to avoid the risk of infection from outside contacts. During this period relatives and friends visit the mother but often are not given the chance to see the new baby. After 40 days, however, the mother is expected to visit all her relatives, taking the baby with her in its new clothes, and she may be too shy to breast feed it outside her new home.

An English health visitor or doctor visiting the new baby may inadvertently interpret these points as signs of child neglect or even child abuse. Such a health worker should not hesitate to ask the mother tactfully for an explanation and should not insist on her bringing the baby to the clinic before six weeks are up as long as the mother and baby are found to be well at home visits. - BASHIR QURESHI, general practitioner, Hounslow, London.

\section{YEARS AGO}

We have before now had to call attention to the difficulty which medical practitioners frequently experience in recovering their fees in cases where they have been retained to attend a confinement, but where their services have not been actually required and rendered. We have pointed out that the right to recover fees in such cases depends on contract, and that the practitioner has no legal claim for fees in cases not actually attended, unless, at the time of being retained, he has stipulated for a fee in any event; but that, if he has so stipulated, and has held himself in readiness to attend, he is morally and legally entitled to be paid. A decision given recently by his Honour Judge Jordan at the Leek County Court, in a case of Bluett $v$ Bryan, if right, would show that fees in cases not actually attended are not recoverable at all; but, as reported, the decision seems thoroughly wrong. Dr. Bluett, the plaintiff, was retained to attend the defendant's wife in her confinement, and when entering the engagement in his book informed her that his fee would have to be paid whether he attended or not. His services were not required, and the defendant declined to pay. The judge decided for $V$ the defendant, on the ground that the Medical Acts prevent a contract in futuro, and that the plaintiff had not rendered any services for which the Acts? authorised him to claim. The report before us is very short, and does not state the sections on which his Honour relied; and we are unable to find any which support his judgment. Section 32 of the Act of 1858 prohibits $\odot$ unregistered persons from recovering charges for "any medical or surgical. advice, attendance, or for the performance of any operation, or for any medicine which he shall both have prescribed and supplied;" and thereby, no doubt, impliedly authorises registered practitioners to recover charges for $\supset$ the services there set out. But neither it, nor any other section of which we are aware, prohibits registered practitioners from entering into contracts and having them enforced in courts of law. Section 6 of the Act of 1886 says that $\varrho$ "a registered medical practitioner shall......be entitled to practise medicine, $\overrightarrow{\overrightarrow{7}}$ surgery, and midwifery ......and to recover in due course of law in respect of $\exists$ such practice any expenses, charges in respect of medicaments or other $\bar{z}$ appliances, or any fees to which he may be entitled." These words seem sufficiently wide to include the case before Judge Jordan, but possibly he was : not aware of the section, as it only came into force in June this year. Apart from it, we think, on the facts as reported, the plaintiff made out his case and was entitled to succeed.

The difficulty of getting a rule established as to payment of fees in midwifery cases is that the amount of fees due in any particular case is small, and can only be sued for in a county court, from which there is no appeal except by leave of the judge, who is pretty sure to refuse it, if he doubts the correctness of his judgment. One judge allows such fees, while in the $\supset$ neighbouring district judgments are given for the defendants. It may be $N$ worth while for the profession to take up a test case, and try and get a decision of the High Court on appeal. But, if so, the case should be carefully selected and got up, and the plaintiff should be properly represented in the county court, otherwise the result may be disastrous. (British Medical 0 foumal 1887; ii:76.)

The number of fatal accidents which have happened on the Swiss Alps this season is already unusually large. During the four weeks ending in the middle of this month, no less than twenty-two persons have thus lost their lives. The majority of the accidents have happened on the lower summits, and are probably all to be traced to the carelessness or inexperience of $\frac{\mathbb{D}}{\mathbb{D}}$ tourists, who are often inclined to believe that because a mountain is not $\stackrel{P}{P}$ covered by perpetual snow, it must be entirely free from danger; this is far $\stackrel{\mathbb{Q}}{2}$ from being the case, and even experienced amateur mountaineers have lost $\bar{O}$ their lives by climbing a "mere alp" (mountain pasture), when not properly equipped for the task; when wearing, for instance, ordinary walking shoes, $\frac{0}{0}$ instead of the stout boots well provided with nails, without which nobody should ever leave the beaten track in a mountainous country. (British $\frac{0}{7}$ Medical foumal 1887;ii:477.) 